Biosensors and Bioelectronics, Volume 20, Issue 2, Special Issue in Honour of Professor Pierre Coulet, 15 September 2004, Pages 145-152

\title{
Surface plasmon resonance sensor for domoic acid based on grafted imprinted polymer.
}

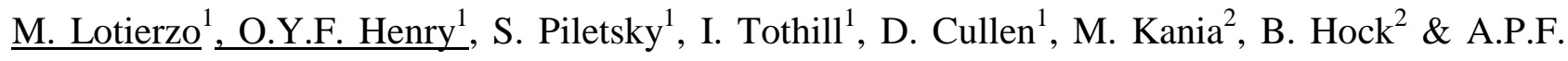
Turner $^{1}$

(1) Institute of BioScience and Technology, Cranfield University, Silsoe, Bedfordshire, MK45 4DT, $U K$

(2) Department of Plant Sciences, Alte Akademie 12, 85350 Freising Germany

\section{Abstract}

A molecularly imprinted polymer (MIP) film for domoic acid (DA) was synthesised by direct photo-grafting onto a gold chip suitable for a surface plasmon resonance (SPR) based bioanalytical instrument system, the BIAcore $3000^{\mathrm{TM}}$. The gold surface was first functionalised with a selfassembled monolayer of 2-mercaptoethylamine and subsequent carbodiimide chemistry was performed for covalent attachment of the photoinitiator, 4,4'-azobis(cyanovaleric acid). This ensured that the formation of the MIP thin film, comprising 2-(diethylamino) ethyl methacrylate as functional monomer and ethylene glycol dimethacrylate as cross-linker, occurred only at the surface level. Optimisation and control over the grafting procedure were achieved using contact angle measurements and atomic force microscope (AFM) imaging. The surface grafting resulted in the formation of thin and homogeneous MIP film with thickness of $40 \mathrm{~nm}$. A competitive binding assay was performed with free DA and its conjugate with horseradish peroxidase, which was used as a refractive label. The sensor was evaluated for its sensitivity, cross-reactivity, and robustness by using a BIAcore $3000^{\mathrm{TM}}$. Likewise, monoclonal antibodies acting as natural receptors for the toxin were studied with the same BIAcore system. Results of a comparison between the artificial and natural receptors are reported. In contrast to monoclonal antibodies, the regeneration of MIP chip 
did not affect its recognition properties and continuous measurement was possible over a period of at least two months.

Keywords: Domoic Acid, MIP, thin films, SPR, AFM.

\section{Introduction}

Domoic acid (DA) is a water-soluble, natural excitatory and neurotoxic amino acid, previously isolated from two red algae: Chondria armata and Alsidium coralliu. This toxin can also originate from marine diatoms (such as Nitzchia pungens) and accumulate in mussels (such as Mytilus edulis), crabs or anchovies. On ingestion by humans, DA contaminated shellfish may cause an intoxication syndrome known as amnesic shellfish poisoning (ASP). The ASP syndrome is characterised by gastro-intestinal symptoms such as vomiting, cramps, and diarrhoea and by neurological symptoms including severe headache, seizures and either temporary or permanent memory loss (Falconer, 1993).

Several analytical methods have been developed for the quantitative determination of domoic acid in shellfish and marine phytoplankton. The original method used for the detection of this toxin was the mouse bioassay, involving intra-peritoneal injection of mice with an acidic extract of whole mussels. A positive effect produces a characteristic syndrome in mice, that is distinct from other marine toxins (Iverson, et al., 1989). A sensitive liquid chromatographic-mass spectrometry (LCMS) method has also been developed for the determination of DA in various marine biological samples (Furey, et al., 2001). Applications of the assay to analyse domoic acid in seawater and shellfish has been also reported both based on antibodies (Kania and Hock, 2002, Smith and Kitts, 1994) and cloned glutamate receptor.

Nevertheless, problems of high cost of measurement and poor stability of biological materials used in assays remains a major obstacle. The ideal systems for the measurement of such toxins should be 
sensitive, robust and relatively inexpensive. Molecular imprinted polymer (MIP) technology is an alternative to natural material, where the cost of imprinting is dictated only by the cost of the template. Since many toxins, including domoic acid, are very expensive, it was necessary to find a way to minimise the amounts of the polymer used in sensor fabrication. Another issue in the fabrication of sensitive evanescent wave-based optical biosensors is related specifically to the extent of the evanescent field, which is only capable of detecting events taking place 100s of $\mathrm{nm}$ above the surface.

In this work we combine recognition elements based on stable and inexpensive ultra-thin MIP films with a sensitive transducer based on surface plasmon resonance (SPR) to develop an optical MIPbased biosensor selective towards domoic acid. By immobilising a photo-labile radical initiator to the gold surface prior to the introduction of the pre-polymerisation mixture, the polymerisation reaction could be confined to the vicinity of the gold surface, resulting in a covalently attached MIP nano-film.

A similar approach was used in a recent work where the technique of grafting polymerization has been used for preparation of thin films of molecularly imprinting polymers on the surface of polypropylene membranes and on hydrophobised gold electrodes for the herbicide desmetryn (Panasyuk-Delaney, et al., 2001). Using another approach Schweitz reported on a novel preparation protocol for MIP film coatings in capillary columns (Schweitz, 2002). The MIP coatings were synthesised 'in situ' under conditions that allow a wide variability of the MIP composition. By immobilising a photo-labile radical initiator to the capillary surface prior to the introduction of the pre-polymerisation mixture, the polymerisation reaction was confined to the vicinity of the capillary surface, resulting in a covalently attached MIP coating. The use of different porogens during the polymerisation reaction facilitates control of the MIP coating in term of morphology and appearance. The MIP coatings synthesised using solvents such as toluene, dichloromethane, and 
acetonitrile, all provided enantiomer separation of the amino alcohol propranolol. In addition, Piletsky et al. (Piletsky, et al., 2000) developed a general method for grafting MIPs to the surface of a stable synthetic polymer. Microfiltration membranes made of polypropylene were used as matrix. Surface photografted copolymerisation using benzophenone as photoinitiator in the presence of template desmetryn introduces specific binding sites into porous membrane without damaging its pore structure and thus preserving its transport properties.

The above approaches require small amounts of polymer and this makes sensor fabrication feasible even for expensive target molecules such as toxins.

\section{Experimental section}

\section{$\underline{\text { Materials }}$}

All reagents and solvents were obtained from Sigma-Aldrich (Poole, Dorset, UK). Domoic acid was purchased from Affinity Research Product Ltd. (Exeter, UK) and the anti-domoic acid antibody was provided by the Technical University of Munich, Germany. Glass slides are from BDH (Poole, Dorset, UK). All solvents were HPLC grade.

\section{$\underline{\text { Vacuum deposition of gold on glass surface }}$}

In order to optimise the photo-grafting procedure the different steps involved were studied by working on a gold-coated glass slide before transferring the MIP film procedure onto the BIAcore chip. To obtain flat gold surfaces, a $1 \mathrm{~cm}^{2}$ glass slide was firstly cleaned of organic contaminants using the following procedure: (i) ultrasonication in deionised (DI) water for 10 minutes, (ii) immersion in $\mathrm{HCl} 5 \mathrm{M}$ for 10 minutes followed by (iii) rinsing in DI water with swirling and subsequent ultrasonication in fresh DI water for $15 \mathrm{~min}$, (iv) ultrasonication in isopropanol for 15 min and finally drying in the stream of argon. The slide were then placed in an Edwards coating 
system E306A (Crawley, UK) and coated under high vacuum $\left(1.5 \times 10^{-6} \mathrm{mbar}\right)$ by evaporation of (i) a $1 \mathrm{~nm}$ thick adhesive chromium layer and (ii) a $50 \mathrm{~nm}$ thick gold layer, which mimics the conditions necessary for the creation of surface plasmon. The sensor chip (Pioneer J1, BIAcore AB, Uppsala, Sweden) consisted of a glass slide mounted in a plastic frame. On one side of the glass, a thin film, approximately $50 \mathrm{~nm}$, of gold had been deposited. Before use, the BIAcore gold chips used for the grafting experiments were cleaned by ultrasonication in ethanol for 30 minutes.

\section{Polymerisation procedure.}

The gold chips were then coated with a self-assembled monolayer of 2-mercaptoethylamine by soaking in $1 \mathrm{mM} 2$-mercaptoethylamine in ethanol for 24 hours at room temperature. Following a brief washing with water and ethanol, the gold surface was again ultrasonicated for 2 minutes in ethanol and dried in argon. The gold chip was coated for 18 hours (in the dark at room temperature) with $1 \mathrm{ml}$ of a solution comprising: $20 \mathrm{mM}$ 4,4'-azo-bis (cyanovaleric acid) photo-initiator, $20 \mathrm{mM}$ water-soluble carbodiimmide and $20 \mathrm{mM}$ 1-hydroxybenzotriazole in ethanol. After another washing step with ethanol, ultrasonication for 2 minutes and drying with argon, $50 \mu$ l of polymerisation solution in water $\left(0.25 \mathrm{mmol}\right.$ of 2 -(diethylamino) ethyl methacrylate, $0.5 \mathrm{mmol}$ of $\mathrm{N}, \mathrm{N}^{\prime}-$ methylenebisacrylamide and $16 \mathrm{nmol}$ of template DA) was purged with argon for five minutes and deposited on top of the gold chip. The mixture on the chip was irradiated with ultraviolet light (UVlamp model $\mathrm{N}^{\circ}$ LX300F, Cermax collimated xenon arc lamp, ILC Technology, Inc., USA) for 30 minutes. After polymerisation, the grafted gold chip was incubated in methanol for two hours in order to remove some homopolymer, residual chemicals and template. Two control chips were grafted following the same procedure described above but without the template domoic acid. A schematic representation of the grafting procedure is presented in Figure 1.

Figure 1: Scheme of the grafting procedure. 


\section{Characterisation of Photo-Grafted MIP by static contact angle measurements}

Photo-grafting of the MIP thin films onto the treated gold surfaces (for SPR studies) was first investigated using contact angle measurements. Contact angle measurements were made using a CCD camera purchased from Spectra Source Equipment (model MCD400S, Westlake Village, California, USA) together with the software provided. Calculations and image processing were made using Paint Shop Pro 5 (Jasc Software). To follow the different changes in surface characteristics due to the various chemical treatments, static contact angle measurements were performed. A $10 \mu \mathrm{L}$ drop of DI water was carefully deposited onto the substrate and images were acquired using the CCD camera.

\section{Atomic Force Microscopy (AFM) Imaging}

Atomic Force Microscopy images were obtained using the PicoScan ${ }^{\mathrm{TM}}$ instrument (Molecular Imaging Corporation, Tempe, AZ, USA). AFM images were taken by scanning the sample relative to a sharp probing tip (which is attached to a cantilever spring) and measuring the deflection of the cantilever as a function of lateral position. The area studied was continuously scanned for one hour (approximately 50 scans). After the additional force was removed, the tip of the cantilever was slightly lifted and moved to scan a larger area $(6 \mu \mathrm{m}$ by $6 \mu \mathrm{m})$. The tip was re-engaged with the surface and the feed back loop re-set. From the topographical image it was possible to evaluate the MIP layer thickness using the software controlling the microscope, which allowed such calculation.

\section{$\underline{\text { MIP Characterisation by BIAcore Analysis }}$}

The BIAcore $3000^{\mathrm{TM}}$ (Biacore $\mathrm{AB}$, Uppsala, Sweden) was used for grafted polymer testing and comparison with monoclonal anti-domoic acid antibody, provided by Prof. Hock from the Technical University of Munich (Germany). The grafted MIP sensor was inserted into the instrument with the gold side in contact with the flow cell. Running buffer (HBS buffer, 10mM 
HEPES, $150 \mathrm{mM} \mathrm{NaCl}, 3.4 \mathrm{mM}$ EDTA and 0.005\% (v/v) surfactant P20, pH 7.4) was constantly passed through the chip at a flow rate of $15 \mu \mathrm{l} \cdot \mathrm{min}^{-1}$ and the temperature was maintained at $25{ }^{\circ} \mathrm{C}$. A steady baseline in the sensorgram curves was achieved (average time 30 minutes to1 hour).

The interaction analysis using BIAcore $3000^{\mathrm{TM}}$ was carried out by injecting $80 \mu \mathrm{l}$ of the conjugate DA-HRP (conjugation ratio 1:1) or native HRP (both provided by Prof. Hock from Technical University of Munich), at concentrations ranging from 6 to $400 \mathrm{mg} \cdot \mathrm{1}^{-1}$ and at a flow rate of $30 \mu \mathrm{l}$. $\min ^{-1}$. The competitive reaction was performed by injecting $150 \mu \mathrm{l}$ of a solution containing an equal volume of HRP-DA conjugate (200 mg. $\left.\mathrm{l}^{-1}\right)$ and the target analytes at different concentration (0-100 $\mu \mathrm{g} \mathrm{l}^{-1}$ ) at flow rate of $30 \mu \mathrm{l} \cdot \mathrm{min}^{-1}$. Each injection was followed by a washing phase with HBS-EP buffer (two injections of $50 \mu \mathrm{l}$ at flow rate of $50 \mu 1 . \mathrm{min}^{-1}$ ) and one regeneration step comprising one injection of $10 \mu \mathrm{l} 0.2 \%$ SDS in $10 \mathrm{mM} \mathrm{HCl}, \mathrm{pH} 2.5$ and at flow rate of $5 \mu 1 . \mathrm{min}^{-1}$. Response units were calculated as the difference in response between two sequential data points in the sensorgram. Cross reactivity (CR) for the grafted polymer was also evaluated for the DA analogues kainic acid (KA), glutamic acid (Glu) and aspartic acid (Asp). The values of \% CR are summarised and compared to anti-domoic acid monoclonal antibody performance (Table 2).

\section{Biacore Analysis of Anti-Domoic Acid Antibody}

The carboxylated dextran matrix of a gold chip CM5 (research grade CM5, Biacore AB, Uppsala, Sweden) was activated by mixing equal volumes of $0.2 \mathrm{M}$ N-ethyl-N'-(3-dimethylaminopropyl) carbodiimide hydrochloride (EDC) and 0.05 M N-hydroxysuccinimide (amine coupling kit, Biacore AB, Uppsala, Sweden). The mixture was injected (35 $\mu \mathrm{l})$ onto the sensor surface for 7 minutes at a flow rate of $5 \mu 1 . \mathrm{min}^{-1}$. The anti-domoic acid antibodies, were firstly immobilised on the chip, in 10 $\mathrm{mM}$ acetate buffer $\mathrm{pH}$ 5. The immobilisation injection flow rate was $5 \mu \mathrm{min}^{-1}$ with a total volume of $35 \mu \mathrm{l}$ for each sample at $25{ }^{\circ} \mathrm{C}$. The procedure was completed by a 7-min injection of $1 \mathrm{M}$ 
ethanolamine hydrochloride $(35 \mu \mathrm{l})$, in order to block any remaining ester groups. One channel was used as a control with bound non-specific antibody (Hock, et al., 2002). Since the immobilised antibody lost a significant amount of its functionality when the regeneration steps were performed, a format with the conjugate immobilised was also investigated. Three different concentrations of the conjugate HRP-DA $\left(150,300,500 \mathrm{mg} . \mathrm{l}^{-1}\right)$ were immobilised by injecting a total volume of $35 \mu \mathrm{lof}$ each sample at flow rate was of $5 \mu 1 . \mathrm{min}^{-1}$ and temperature of $25^{\circ} \mathrm{C}$. The conjugate surface was normally regenerated with a 1 min injection of regeneration buffer (SDS $0.2 \%-\mathrm{HCl} 10 \mathrm{mM}, \mathrm{pH}$ 2.5). To avoid the problem of regenerating the antibody bound to the sensor chip, an assay format with the bound conjugate DA-HRP was investigated. The conjugate (150 mg. $\left.\mathrm{l}^{-1}\right)$ was covalently immobilised to the dextran matrix and served as an affinity surface. Free protein ovalbumin was immobilised onto a separate channel and was used as a control surface. The anti-domoic acid antibody $\left(6.6 \mu \mathrm{g} \cdot \mathrm{ml}^{-1}\right)$, together with different concentrations of free domoic acid, was injected in a volume of $35 \mu \mathrm{l}$ and at a flow rate $5 \mu \mathrm{l} \cdot \mathrm{min}^{-1}$. Binding and regeneration conditions were optimised in a separate set of experiments (data not shown).

\section{Results and discussion}

Although molecular recognition sensors based on molecularly imprinted polymer as recognition elements have been described in the past, they often rely on bulk polymers ground to desired particle sizes and subsequently immobilised on the transducer surface by different means. Unfortunately, this approach cannot be used if the cost of the template is too high (as in the case of domoic acid). The problem can be overcome by using a grafting procedure, which requires minimal quantities of the analyte to synthesise a thin polymer film in the surface of the transducing element. The grafting procedure also aids in solving problems related to integrating MIPs with transducer as homogenous ultra-thin films, especially for SPR detection. The photo-grafting procedure in principle is ideally suited for this task since it provides the possibility to control the deposition rate by controlling the time and intensity of UV exposure. 


\section{Polymer Grafting}

The various steps involved in the grafting of a thin MIP layer were inspired by the techniques used in peptide coupling and protein synthesis. Having found a potential photo-initiator with symmetrical carboxylic acid groups at each arm, it was possible to envisage a covalent attachment of the polymerisation initiator to the amino-functionalised gold surface through carbodiimide chemistry. Over the past decade, design of the optimal solvent mixture for peptide coupling and protein synthesis has been extensively studied. Nozaki (Nozaki, 1999, Nozaki, 1997) showed that the coupling yield of Boc-Ala-OH and H-Gly-Obz-L in equimolar quantity of HOBt and EDC was $100 \%$ after 16 hours in pure DMF. This approach was therefore adjusted and employed successfully in this work where a mixture of EDC, HOBt, and 4,4'-azo-bis (cyanovaleric acid) in DMF were allowed to stand-alone for one hour in order to activate the carboxylic groups of the photo-initiator. After this activation step, the amino-functionalised gold surface was introduced into the "coupling" reaction mixture. After 16 hours in the dark, the covalent attachment of the photo-initiator to the surface had taken place. The ultrasonication did not remove the covalently attached initiator and did not reduce the quality of the final polymer coating obtained.

\section{Characterisation of Grafting Technique by Static Contact Angle measurements and AFM Imaging}

Two techniques were used for the characterisation of grafted films: contact angle measurements and atomic force microscopy (AFM). Table 1 presents the results of the contact angle measurements. Each step in the chemical derivatisation of the surface induced a change in the hydrophobic/hydrophilic properties of the surface. The clean bare gold substrate was very hydrophilic and showed angles less than $20^{\circ}$, whereas once derivatised with 2-mercaptoethylamine the angle changed radically to $43^{\circ}$. These values were in agreement with the published data on performance of similar systems (Tsukruk, et al., 1998). The ensuing grafting of the MIP layer showed another variation in the contact angle at $56^{\circ}$. 
Table 1: Static contact angle measurements

AFM images were obtained using contact mode scanning. These measurements provided a method of following the chemical modification step. Figures 2 A and $\mathbf{B}$ show the topographical characteristics of a $2 \mu \mathrm{m}$ by $2 \mu \mathrm{m}$ area of the MIP layer deposited. The general features have a maximum differential height of $13 \mathrm{~nm}$, which confirm the homogeneity of MIP films photo-grafted onto gold. The "scrapping" experiment was performed as described in Experimental section.

Figure 2A and 2B: Planar (a) and 3D (b) topographic image of a gold chip with grafted MIP.

Figure 3 presents the topographic (also called Z-image) on the left and the deflection images of a larger area (right). The horizontal line allows a horizontal cross section profile to be drawn of the surface as is depicted below each image. From the topographical image, it is possible to evaluate the MIP layer thickness.

Figure 3: $6 \mu \mathrm{m} \times 6 \mu \mathrm{m}$ AFM image of a $2 \mu \mathrm{m} \times 2 \mu \mathrm{m}$ scraped area of a MIP grafted chip and the cross section analysis (below).

The left image in Figure 3 shows a neat "cuvette" corresponding to the scrapped area. The difference between the two levels could be calculated from the $\mathrm{Z}$ position of the two cursors 1 and 2 , representing the levels of the un-scrapped and scrapped areas, respectively. In order to evaluate the overall height difference between the two levels, we plotted the colour histogram in Figure 4, which give us a better evaluation of the polymer thickness. The low and high peaks represent the scrapped and un-scrapped areas respectively. The deflection image (right image on Figure 3) shows also how the texture of the scrapped area has completely changed, revealing the bare gold 
underneath, when compared to the surrounding area covered by polymer. The thickness calculated from the topographic image is representative of the layer deposited.

Figure 4: AFM histogram.

\section{$\underline{\text { SPR Study of Polymer-Domoic Acid Interactions }}$}

Gold chips coated with MIP and blank polymers were prepared and tested for their capability to recognise domoic acid. Since domoic acid is a small molecule $(\mathrm{FW}=311)$, which does not significantly change refractive index, a conjugate of domoic acid and HRP was used for analysis of polymer specificity. Either the conjugate DA-HRP or HRP were injected into the BIAcore $3000^{\mathrm{TM}}$ $(80 \mu \mathrm{l})$ with a flow rate of $5 \mu 1 \cdot \min ^{-1}$ (Figure 5).

Figure 5: Specific binding of DA-HRP conjugate to grafted MIP compared to non-specific binding of native HRP. Error bars $= \pm S D, n=3$.

The coated MIP chip displayed a specific binding of domoic acid-HRP conjugate, shown by the increase in the response unit (RU) of the chip (RUmax > 450), whereas a much lower signal was observed for native HRP enzyme (RUmax < 100). At a concentration of $200 \mathrm{mg} \cdot \mathrm{l}^{-1}$ of conjugate, the maximum difference (signal/control) with the same concentration of free HRP was equal to 4.7. The concentration of $200 \mathrm{mg} . \mathrm{l}^{-1}$ was subsequently used for the competitive reaction with the MIP optical sensor.

The regeneration was optimised by changing the volume and the flow of the injection as well as the composition of the regeneration solutions. Since the solution used for regeneration differs significantly in refractive index from running buffer, it gives a high positive bulk response. 
A complete regeneration of MIP receptor was obtained after injecting $10 \mu \mathrm{l}$ of $0.2 \%$ sodium dodecil sulphate (SDS) in $10 \mathrm{mM} \mathrm{HCl}$ at a flow rate of $5 \mu \mathrm{min}^{-1}$. A faster flow rate did not give a complete regeneration even using larger volume. The regeneration solution did not appear to harm the polymer receptor sites since after more than 30 regenerations steps with SDS $0.2 \%$ in $\mathrm{HCl} 10 \mathrm{mM}$, a competition reaction was still observed on the coated MIP and no loss of signal was observed (Figure 6).

Figure 6: Two consecutive regeneration phases alternated with one injection of the analyte.

The baseline level after regeneration should ideally be identical to that before the sample injection. Provided that the baseline is restored to the correct level when the response has stabilized, this did not affect the kinetic determinations. A constant flow system running the PBS buffer containing $0.05 \%$ Tween 20 at $30 \mu 1 . \mathrm{min}^{-1}$ facilitated washing away of the weak non-specific interactions. The competitive reaction was carried out by injecting into the flow cell of the BIAcore $3000^{\mathrm{TM}}$ system an equal volume of conjugate DA- HRP (200 mg. $\left.\mathrm{l}^{-1}\right)$ and a broad range of domoic acid concentrations over a range of 1-100 $\mu \mathrm{g} .1^{-1}$. A regeneration step followed each of the analyte injections; a space of approximately 5 minutes was left between injections. A calibration curve for the competitive reaction on the MIP sensor is shown in Figure 7.

Figure 7: Competitive calibration curve for the domoic acid MIP sensor.

The limit of detection calculated as an $\mathrm{LLD}_{80}$ corresponding to $5 \mu \mathrm{g} . \mathrm{l}^{-1}$ and the $\mathrm{EC}_{50}$ value was 58 $\mu \mathrm{g} .1^{-1}$. The response curve is highly reproducible, as indicated by the relatively low standard deviations and the mean \% CV for the assay. 
The cross reactivity (CR) of the grafted polymer for the DA analogues kainic acid (KA), glutamic acid (Glu) and aspartic acid (Asp) was also evaluated. Injections of equal volumes (150 $\mu \mathrm{l})$ of HRPDA conjugate and the target analytes $\left(0\right.$ and $\left.5 \mu \mathrm{g}^{-1}\right)$ were introduced to the MIP sensor at flow rate of $30 \mu \mathrm{min}^{-1}$. The values of $\%$ cross-reactivity are summarised and compared to anti-domoic acid monoclonal antibody performance in Table 2 .

The stability of the polymer coating was analysed by storing gold chips at $4{ }^{\circ} \mathrm{C}$ for a period of up to three months and by regular measurement of the sensor signal. A summary of the two receptor's performances for DA detection is presented in Table 2.

Table 2: Comparison between MIP and anti-DA antibody.

\section{Conclusions}

Artificial MIP receptors represent an attractive alternative to antibodies since they can perform similarly to natural macromolecules, but provide better stability and in general are inexpensive to prepare. In the present paper, a new MIP receptor was designed for domoic acid and used for development of an evanescent (SPR) sensor for this compound. Atomic force microscopy (AFM) analysis and contact angle analysis showed the resultant grafted MIP layer to be approximately 40 $\mathrm{nm}$ thick and deposited homogeneously; it was thus suitable for SPR-based analyses. In term of sensitivity, the MIP had approximately three times higher detection limit $\left(\operatorname{LLD}_{80}\right)$ compared to that of monoclonal antibody (5 $\mu \mathrm{g} \mathrm{l}^{-1}$ versus $1.8 \mu \mathrm{g} \mathrm{l^{-1 }}$ of domoic acid respectively). The calibration curve for the MIP sensor showed a maximum value of approximately 400 RU, which was half of the maximum RU value observed for the antibody. However, the detection range of the MIP sensor was considerably improved (5-100 $\left.\mu \mathrm{g}^{-1}\right)$ compared to the immunosensor. This was due to the varied distribution of the polymer binding sites, which presented a variety of different affinities for the target molecule, as previously discussed by Mosbach (Mosbach and Ramstrom, 1996). 
When directly immobilised on the gold surface, the antibodies lost much of their activity after a few regeneration cycles. In contrast to the antibodies, MIPs appeared to be robust enough to withstand harsh treatment and multiple regeneration cycles.

\section{Acknowledgements}

We gratefully acknowledge the financial support of the European Union, Environmental and Climate programme (EN V4-CT98-0784).

Sergey Piletsky would like to acknowledge with gratitude receiving the Royal Society-Wolfson Research Merit Award.

\section{References}

Falconer, I. R., 1993. Paralytic Shellfish Poisons from Fresh-Water Blue-Green-Algae - Comment. Medical Journal of Australia 159(6), 423-423

Furey, A., M. Lehane, M. Gillman, P. Fernandez-Puente and K. J. James, 2001. Determination of domoic acid in shellfish by liquid chromatography with electrospray ionization and multiple tandem mass spectrometry. Journal of Chromatography A 938(1-2), 167-174

Hock, B., M. Seifert and K. Krämer, 2002. Engineering receptors and antibodies for biosensors. Biosensors \& Bioelectronics 17,239-249

Iverson, F., J. Truelove, E. Nera, L. Tryphonas, J. Campbell and E. Lok, 1989. Domoic Acid Poisoning and Mussel-Associated Intoxication - Preliminary Investigations into the Response of Mice and Rats to Toxic Mussel Extract. Food and Chemical Toxicology 27(6), 377-\&

Kania, M. and B. Hock, 2002. Development of monoclonal antibodies to domoic acid for the detection of domoic acid in blue mussel (mytilus edulis) tissue by ELISA. Analytical Letters 35(5), 855-868

Mosbach, K. and O. Ramstrom, 1996. The emerging technique of molecular imprinting and its future impact on biotechnology. Bio-Technology 14(2), 163-170 
Nozaki, S., 1999. Effects of amounts of additives on peptide coupling mediated by a water-soluble carbodiimide in alcohols. Journal of Peptide Research 54(2), 162-167

Nozaki, S., 1997. Efficient amounts of additives for peptide coupling mediated by a water-soluble carbodiimide in aqueous media. Chemistry Letters 1,1-2

Panasyuk-Delaney, T., V. M. Mirsky, M. Ulbricht and O. S. Wolfbeis, 2001. Impedometric herbicide chemosensors based on molecularly imprinted polymers. Analytica Chimica Acta 435(1), $157-162$

Piletsky, S. A., H. Matuschewski, U. Schedler, A. Wilpert, E. V. Piletska, T. A. Thiele and M. Ulbricht, 2000. Surface functionalization of porous polypropylene membranes with molecularly imprinted polymers by photograft copolymerization in water. Macromolecules 33(8), 3092-3098 Schweitz, L., 2002. Molecularly imprinted polymer coatings for open-tubular capillary electrochromatography prepared by surface initiation. Analytical Chemistry in press),

Smith, D. S. and D. D. Kitts, 1994. A competitive enzyme-linked immunoassay for domoic acid determination in human body fluids. Food and Chemical Toxicology 32(12), 1147-1154

Tsukruk, V. V., Z. Huang, S. A. Chizhik, V. V. Gorbunov and N. K. Myshkin, 1998. Dynamic probing of micromechanical properties of polymeric materials. Abstracts of Papers of the American Chemical Society 216,239-PHYS 